\title{
Thermostatic and Dynamic Performance of an Ultrasonic Density Probe
}

\author{
Jan van Deventer and Jerker Delsing, Member, IEEE
}

\begin{abstract}
The thermally static and dynamic performance of an ultrasonic density probe for liquids is investigated in the density range of 750 to $1300 \mathrm{~kg} / \mathrm{m}^{3}$ at temperature ranging from 0 to $40^{\circ} \mathrm{C}$. The single transducer probe uses a pulse echo technique to obtain the characteristic acoustic impedance of the liquid and, subsequently, the speed of sound through the liquid to obtain the density of the liquid. Variations in the initial sound amplitude are addressed by the design of a layered two material probe. It is shown that it is possible to obtain an accuracy of $0.4 \%$ in the experiments carried out. For changing temperature, the probe exhibits large errors because of problems in estimating the temperatures in certain regions of the probe.
\end{abstract}

\section{INTRODUCTION}

$\mathrm{U}$ NMONITORED fluctuations in the density of a flowing liquid can adversely affect both product quality and cost assessment in industry. Consider the energy industry, where energy is being transferred via a liquid. This liquid can be a fuel or simply hot water as in a district heating system. Measurement of the amount of liquid transferred in terms of volume is erroneous when the density of the liquid changes. In this case, the measurement of mass flow is more appropriate than that of volumetric flow. Variations in liquid densities arise from thermal expansion or changes in the liquid's constituent parts. Inaccurate cost assessment caused by improper flow measurement results in very large sums for the industries, costs that are ultimately born by the end users.

There are different methods of measuring mass flow, but the use of ultrasonic instruments can offer the best solution in certain applications. Compared with direct mass flow measuring devices, an ultrasonic mass flow meter is insensitive to the vibrations that affect a Coriolis mass flow meter and is generally better suited for use with liquids than is a thermal mass flow meter [1]. The ultrasonic mass flow meter belongs to the category of indirect mass flow meters. In this category, one measures volumetric flow and density separately and combines the results to obtain a mass flow estimate. Ultrasonic systems have the advantage of being online, non-intrusive, non-radioactive, and potentially lower in cost for an equivalent accuracy.

Complete ultrasonic mass flow measurement systems require an ultrasonic volumetric flow meter and an ultra-

Manuscript received August 16, 1999; accepted October 12, 2000.

The authors are with the Department of Computer Science and Electrical Engineering, Lulea University of Technology, Lulea S97187, Sweden (e-mail: deventer@sm.luth.se). sonic densitometer. The former has been in use for several decades. It calculates the fluid flow velocity from the difference between the up and downstream times of flight of acoustic pulses traveling between two ultrasonic transducers [1]. From the two times of flight, one can additionally calculate the bulk speed of sound through the liquid. The non-intrusive ultrasonic densitometer measures the characteristic acoustic impedance of the liquid. The specific acoustic impedance relates the pressure of the acoustic wave to the particle velocity of the medium. For progressive plane waves, it is then the characteristic acoustic impedance or the product $\rho c$ (with $\rho$ representing the density of the medium and $c$ the bulk speed of sound through the medium) [2]. The density of the liquid is obtained by dividing the measured characteristic impedance by $c$, the by-product of the volumetric flow meter.

Ultrasonic densitometers based on the idea of measuring the characteristic acoustic impedance are not new and their fundamental concepts are interestingly different. In the 1950s, Kritz was awarded patents for ultrasonic mass flow meters that use a continuously oscillating ultrasonic crystal in contact with the liquid to measure acoustic impedance [3], [4]. The principle of operation relies on the fact that the electrical impedance across the crystal is dependent on the acoustic impedance of the liquid in contact with the crystal. Sukatskas presents a closely related acoustic impedance meter by spectrally sweeping the ultrasonic crystal [5]. The resonant frequency and bandwidth of the crystal are correlated to the contacting liquid's characteristic impedance. Sukatskas also shows that the crystal does not need to be in direct contact with the liquid, and the resonating element can then be the intermediate layer separating the liquid and the crystal.

The pulse-echo technique offers a different method to obtain the liquid's acoustic impedance. An acoustic pulse is emitted from an ultrasonic crystal into an intermediate layer toward the liquid. As the acoustic pulse enters the liquid from the intermediate layer, part of its energy is reflected as an echo to the crystal by an amount related to the acoustic impedance of the liquid (Fig. 1). The amplitude ratio $R$ of the echo to the incident pulse normal to the flat interface is

$$
R_{12}=\frac{z_{2}-z_{1}}{z_{1}+z_{2}}
$$

where $z$ is the characteristic impedance of the medium referred to by each of the subscripts. With the amplitude of the incident acoustic pulse, the characteristic impedance of the intermediate layer known, and the amplitude of the 


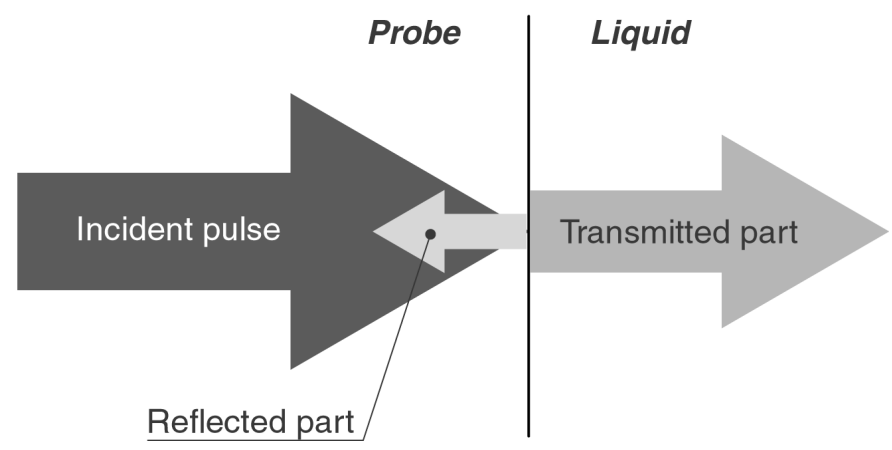

Fig. 1. Reflection and transmission of sound at the interface between two media with different acoustic impedances.

reflected echo measured, one calculates the characteristic impedance of the liquid. This is the method pursued here, as it permits the incorporation of the densitometer into one of the volumetric flow meter's two ultrasonic transducers.

Instruments whose construction is based on the theory presented here function well in a laboratory setting. However, two major problems have slowed the introduction of ultrasonic mass flow meters to the field. The first one has to do with the fact that the amplitude of the emitted pulse can vary. Amplitude variations may be caused by changes in the associated excitation electronics or the aging of the probe's components. If one assumes that the amplitude of the incident pulse is constant, and it is not monitored, then the calculation of the liquid's acoustic impedance might be incorrect. Investigations addressing this issue are presented in the following paragraph. The second problem has to do with the great influence of temperature on many of the probe's characteristics. Characteristic impedance; acoustic attenuation; physical dimensions; and, therefore, the density of the probe are all temperature-dependent. Again, assuming them to be constant leads to an incorrect estimation. The current work concentrates on this issue.

Different schemes have been investigated to monitor the amplitude of the initial pulse. One solution has been the introduction of a receiver in the probe's intermediate layer or buffer rod [6]. A thin PVDF plastic membrane covered by electrodes on each side is inserted into the buffer rod, perpendicular to the direction of sound propagation. It senses the passing planar acoustic waves to determine the amplitude of the initial pulse as a reference as well as the amplitude of subsequent echoes. Another solution involves the addition of a second transducer in parallel [7]. Both transducers emit an acoustic pulse into the measured liquid, which contains a divider. As the sound enters the divider, an echo is reflected back to each transducer. When leaving the divider, the pulse from the first transducer enters the liquid, whereas the pulse from the second transducer encounters air. The latter provides an almost total reflection; the former does not. Based on the amplitude of the four received echoes, one calculates the acoustic impedance of the liquid. A third scheme uses the same probe material in front and in back of the ultrasonic crystal [8]. The back buffer rod, which is terminated in air, provides a nearly

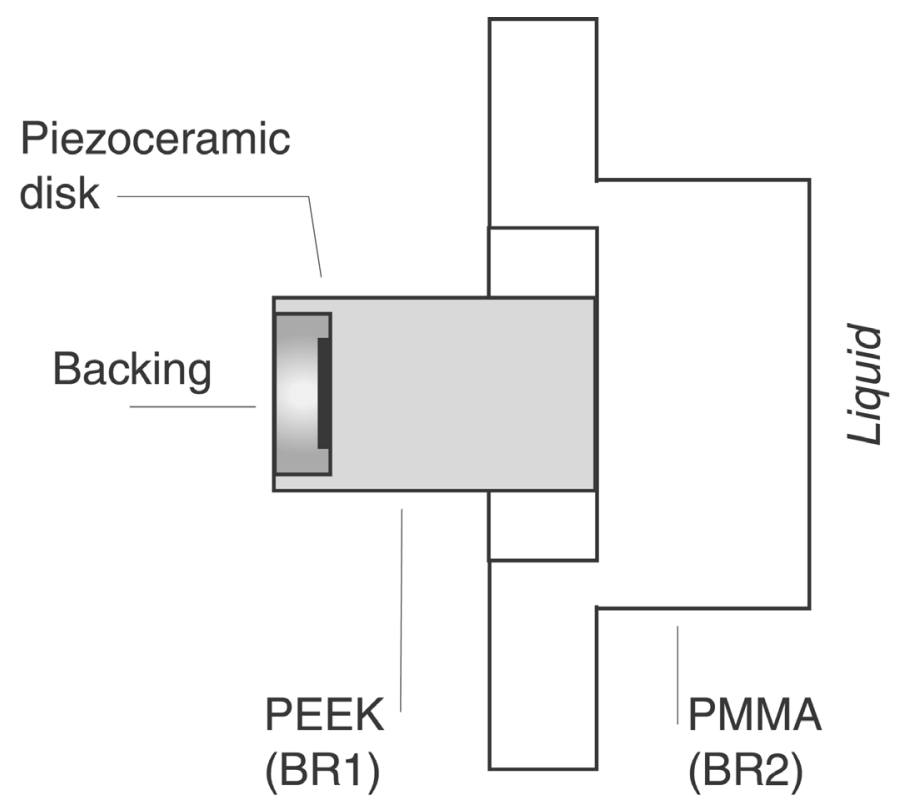

Fig. 2. Cutaway of the ultrasonic density probe.

complete reflection and, thus, a reference echo. Yet another concept involves the insertion of a new buffer rod between the probe's buffer rod and the transducer [9]. The additional interface between two known media provides a reference echo. The present research focuses on this design as an extension of work presented earlier [10]. It is also chosen to support future research in view of its potential in the construction of a compact probe with echoes overlapping in time.

Following this introduction, the probe is described in detail. A section on the signal processing used to extract the necessary information from the recorded sound succeeds the introduction. The focus of the present paper is on the thermal performance of an ultrasonic density probe. The density probe is evaluated in uniform temperatures in the range of 0 to $40^{\circ} \mathrm{C}$ and against liquids with a density of 700 to $1300 \mathrm{~kg} / \mathrm{m}^{3}$. The performance of the densitometer is also investigated while the temperature is changing. In this setting, the thermodynamic and thermal gradient of the probe are presented.

\section{Probe}

The amplitude variation issue of the emitted pulse is addressed by measuring the amplitude of a reference echo. The reference echo is a reflection from an interface separating two known media. Measurement of the echo's amplitude and knowledge of the characteristic impedance of the two media allow the calculation of the emitted pulse's amplitude with the use of (1). The introduction of the new buffer rod (buffer rod 1 or BR1) between the ultrasonic crystal and the buffer rod in contact with the fluid (buffer rod 2 or BR2) presents the needed interface (Fig. 2).

The lattice diagram (Fig. 3) gives a perspective of the sound propagation in space and time. The received signal 


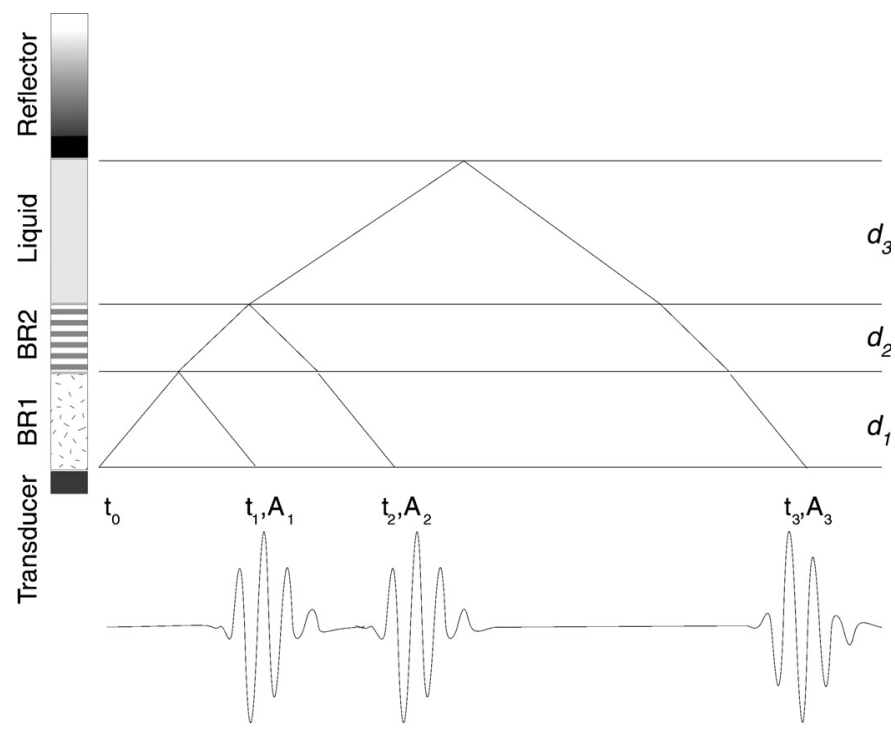

Fig. 3. Lattice diagram with scaled echoes in the received signal.

is shown below the diagram, where each echo is scaled to clarify the figure. The transducer emits a pulse with an amplitude $A_{0}$ at time $t_{0}$. The pulse propagates through $\mathrm{BR} 1$ with speed $c_{1}$. It is exponentially attenuated with respect to the distance traveled $d_{1}$ at the rate $\alpha_{1} / \mathrm{m}$. It is echoed back at the interface with BR2 and is again exponentially attenuated. At time $t_{1}$, the reference echo is received with an amplitude

$$
A_{1}=A_{0} R_{12} e^{-2 d_{1} \alpha_{1}} .
$$

The factor of 2 in the exponential decay term accounts for the back and forth propagation of the pulse. The indices of the coefficient of reflection $R$ refer to the media of BR1 and BR2.

The energy of the pulse not reflected is transmitted into BR2. There, the pulse travels with a speed $c_{2}$ toward the probe liquid interface, where it will be partly reflected. At time $t_{2}$, the echo from that interface is received by the transducer with an amplitude

$$
A_{2}=A_{0} T_{12} R_{23} T_{21} e^{-2 d_{1} \alpha_{1}} e^{-2 d_{2} \alpha_{2}}
$$

where $\alpha_{2}$ is the coefficient of attenuation in BR2, and the index 3 refers to the liquid medium. The coefficient of transmission $T$ is the amplitude ratio of the transmitted pulse to the incident pulse. The first index refers to the transmitting side of the interface; the second refers to the receiving side. In terms of characteristic impedance,

$$
T_{i j}=\frac{2 z_{j}}{z_{i}+z_{j}}
$$

with, again, the requirements of progressive plane waves incident normal to a smooth and flat interface.

The measured amplitude $A_{1}$ can replace the unknown amplitude $A_{0}$ in (3) with the use of (2). Solving for the liquid's characteristic acoustic impedance $z_{3}$ from $(3)$, one obtains

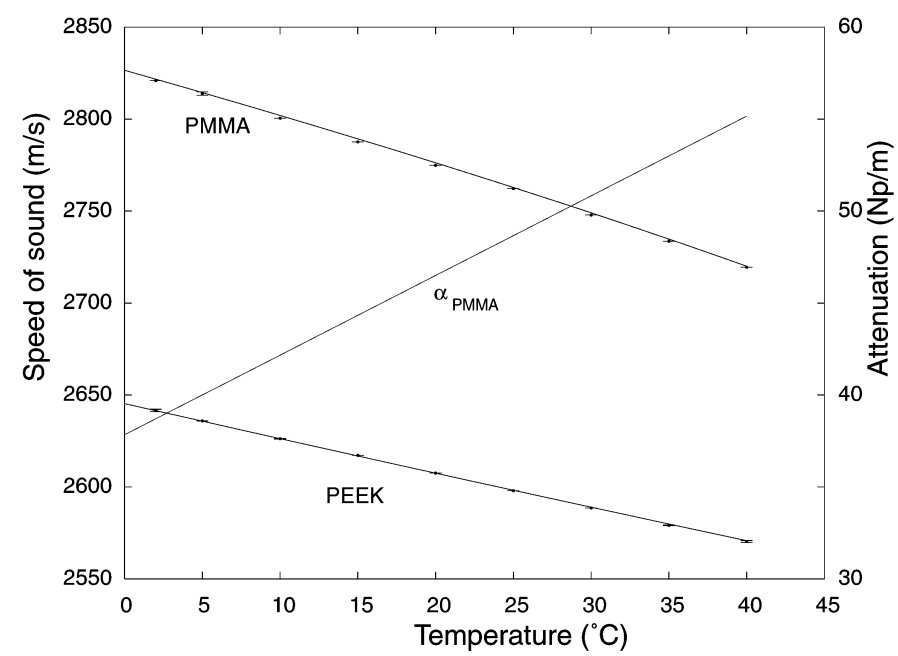

Fig. 4. The acoustic characteristics of the probe's polymers at $2.0 \mathrm{MHz}$.

$$
z_{3}=z_{2} \frac{\left(4 A_{1} z_{1} z_{2} e^{-2 d_{2} \alpha_{2}}-A_{2}\left(z_{1}^{2}-z_{2}^{2}\right)\right)}{\left(4 A_{1} z_{1} z_{2} e^{-2 d_{2} \alpha_{2}}+A_{2}\left(z_{1}^{2}-z_{2}^{2}\right)\right)} .
$$

The bulk speed of sound $c_{3}$ in the liquid remains to be determined to compute the liquid's density. To study the densitometer's performance, the flow meter section described in the introduction has been removed. The densitometer instead listens for an echo of the pulse transmitted in the liquid. The echo comes from a reflector located at a known distance within the fluid. The speed of sound through the liquid is calculated with the time of flight between the second and third echoes:

$$
c_{3}=\frac{2 d_{3}}{\left(t_{3}-t_{2}\right)} \text {. }
$$

With a similar densitometer, Delsing [9] reported a performance with an uncertainty of $0.6 \%$ at a $95 \%$ confidence level. The liquid density ranged from 800 to $1200 \mathrm{~kg} / \mathrm{m}^{3}$ at a constant temperature of $24.0 \pm 0.5^{\circ} \mathrm{C}$. The current probe matches this performance. If the temperature is not taken into account, the error increases to $3 \%$ when the measurement is done $1^{\circ} \mathrm{C}$ away from the calibration's temperature. Any variation in temperature alters the dimensions, and thus the density, speed of sound, and attenuation, in all media. The change in speed of sound, perceptible in the measured time of flight through each medium, is used to estimate the temperature of each buffer rod. The relationships between the speed of sound and the probe's dimensional changes and attenuation as a function of temperature can be established (Fig. 4). In other words, from the times of flight, the temperature of each buffer rod can be estimated and, accordingly, so can the characteristic impedance $z_{1}$ and $z_{2}$ and the attenuation factor $\alpha_{2}$.

The attenuation of sound in BR1, $\alpha_{1}$, does not appear in (5). The fact that any echo returning to the transducer through BR1 undergoes the same exponential decay explains its absence. The sound attenuation $\alpha_{2}$ in BR2 (Fig. 4) has a higher value than the attenuation of sound caused by viscoelasticity in that polymer. It in- 
cludes the frequency-dependent diffraction and the influence of the coupling layer between the two buffer rods. This layer, whose thickness amounts to only a few microns, influences the amplitude measurement of the propagating wave. The cumulative coefficient of attenuation $\alpha_{2}$ is both temperature- and frequency-dependent. All amplitude measurements are done at one frequency to exclude the latter dependency.

\section{A. Probe Construction}

From the associated electronics, the electrical leads are soldered to each side of a 10-mm PZ-27 piezoceramic disc manufactured by Ferroperm (Denmark). With a thickness of $1 \mathrm{~mm}$, it has a fundamental frequency of $2 \mathrm{MHz}$. It is permanently glued to a PEEK (polyetheretherketone) buffer rod (BR1) (Fig. 2) and backed with an acoustically absorbing epoxy. The backing epoxy has a characteristic acoustic impedance of $4.58 \mathrm{MPa} \cdot \mathrm{s} / \mathrm{m}$ and a density of $1600 \mathrm{~kg} / \mathrm{m}^{3}$. The PEEK buffer rod has a diameter of $18 \mathrm{~mm}$ and a length of $25 \mathrm{~mm}$. The long ring down of the transducer requires such length to distinctly separate the echoes in time. BR2, made of PMMA (polymethyl methacrylate), has a diameter of $40 \mathrm{~mm}$ and a length of $20 \mathrm{~mm}$. The two buffer rods are permanently bonded with a cyanoacrylate adhesive because temporary couplants change their characteristics after days of use. The two polymers were glued together at a temperature between 20 and $25^{\circ} \mathrm{C}$. The PEEK plastic has an acoustic characteristic impedance of $3.20 \mathrm{MPa} \cdot \mathrm{s} / \mathrm{m}$, and PMMA has one of $3.19 \mathrm{MPa} \cdot \mathrm{s} / \mathrm{m}$. Their thermal coefficients of expansion are $47 \cdot 10^{-6}$ and $60 \cdot 10^{-6} / \mathrm{K}$, respectively. All of the values given here are referenced to $20^{\circ} \mathrm{C}$.

\section{Signal Processing}

The piezoceramic disk receives the acoustic signal and transforms it into an electrical one. This electric signal goes through amplification and digitization to enable a computer to process it and draw out the needed information. To satisfy (5), two types of information are desired from it: the amplitude of each echo and the times separating them. From the complete signal, the three echoes are located with a trigger level, extracted, and centered into 2048 data point long windows.

\section{A. Frequency Dependent Amplitudes}

Because acoustic attenuation is frequency-dependent, the amplitudes of the echoes are measured in the frequency domain at a single frequency. A fast Fourier transform algorithm (FFT) [11] provides a discrete frequency spectrum of each echo in its respective window. The magnitudes $A_{1}$ and $A_{2}$ of the first two echoes are measured at $2.0 \mathrm{MHz}$, the resonant frequency of the transducer.

The relative phase of $A_{1}$ and $A_{2}$ influence the use of (5). A reflected echo has a $180^{\circ}$ phase shift with its incident and transmitted pulse if the reflecting medium has a lower characteristic impedance as indicated by (1). With the present probe, both $R_{12}$ and $R_{23}$ are negative and in phase with each other. Therefore, there is no need to negate either $A_{1}$ or $A_{2}$.

\section{B. Times of Flight}

The time of flight through each medium is first approximated by the difference between the start of two successive windows. Because the location of the echoes is based on a trigger level, the times of flight are usually accurate to only one oscillation. Locating the occurrence of the maximum value of a cross-correlation between the two windows provides a lead or lag time of the echoes with respect to the windows. Correcting the approximate time of flight by this lead or lag time provides a time of flight accurate to a sampling period.

These cross-correlations are performed in the frequency domain with the discrete Fourier-transformed echoes calculated earlier for the amplitude determination. The inverse FFT will lead back to the time domain. A search for the absolute maximum will indicate the correct time shift. Because the pulse distortion caused by the frequencydependent attenuation is minimal, the cross-correlation method is suitable.

The previously mentioned method cannot measure the time of flight through BR1 because the excitation spike with its ring down and the first echo are too dissimilar. During the probe's design phase, an intermediate buffer rod was placed between the transducer and PEEK samples. The speed of sound through PEEK was measured using the described method. With the actual probe, the time of flight is first estimated as the beginning of the first window $t_{w 1}$. The time of the excitation spike is $t_{0}=0$. This time is shorter because the echo occurs within the window. To obtain a more appropriate estimate, a temperatureindependent correction factor $\delta$ is added to that time. This estimate can easily vary by at least a half period because of the trigger level detection mechanism. Using the crosscorrelation method with a reference signal offers a solution. At a reference temperature of $20^{\circ} \mathrm{C}$, a signal is acquired. Its first window is saved as a reference, and the correction factor $\delta$ is correctly set to provide the correct time of flight for the temperature and dimension of the buffer rod. For any subsequently acquired signal, the time of flight through BR1 is obtained by adding $\delta$ to the start of the first new window. Performing a cross-correlation of the new first window and the reference window and locating the occurrence of its maximum puts forth a lead or a lag time $\tau$. $\tau$ refines the time of flight estimate to a sampling period such that

$$
t_{1}=t_{w 1}+\delta-\tau
$$




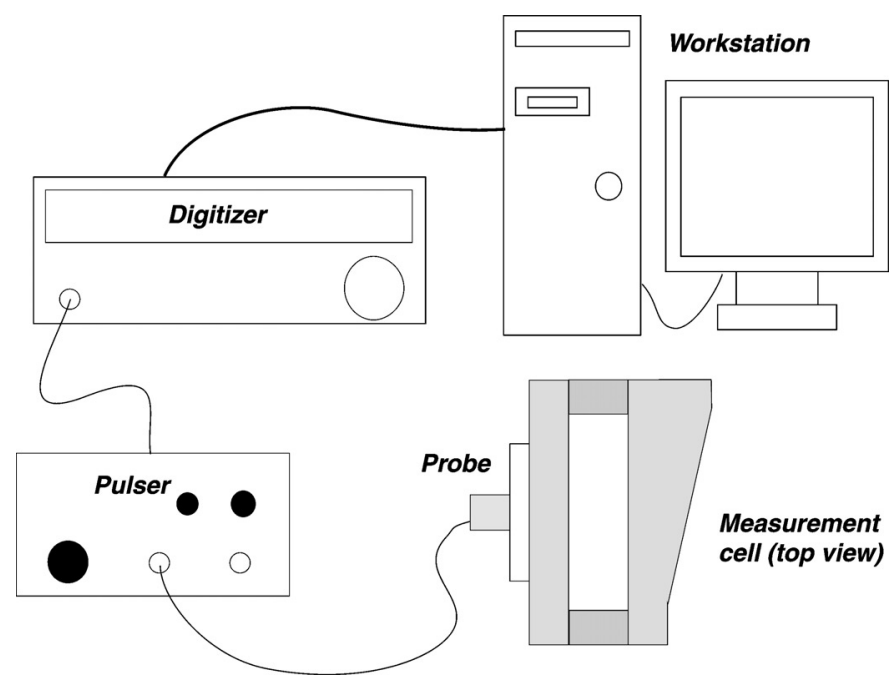

Fig. 5. Experimental setup.

\section{EXPERIMENTS AND RESUlts}

\section{A. Experimental Setup}

The probe is fastened horizontally to the measurement cell. The measurement cell consists of two stainless steel plates separated by two $30-\mathrm{mm}$ brass bars, all of which stands on a Plexiglas bottom (Fig. 5). The back stainless steel plate has a polished surface facing the probe, which acts as a reflector. Its outer surface is rough and angled to dissipate any transmitted energy.

A Heraeus HT4010 temperature test chamber houses the measurement cell and probe. The transducer is connected to a Panametrics 5072PR pulser-receiver located outside the chamber. Amplified by the pulser-receiver, the received analog signal is sampled at $200 \mathrm{MHz}$ by a SonyTektronix RTD 710 digitizer with a 10-bit resolution. Averaged 32 times, the digitized waveform is transferred to a workstation via a GPIB (general purpose interface bus). There, a program reduces the signal to a density measurement. A Systemteknik S2541 thermolyzer, accurate to $0.01^{\circ} \mathrm{C}$, monitors the temperatures of the liquid sample and the measurement cell's environment using two PT100 probes. Every $2 \mathrm{~s}$, the workstation receives new temperature information via an RS232 connection.

\section{B. Calibration}

During the design phase, the thermal expansions of the two polymers were verified on buffer rods. At the same time, the speed of sound in both materials was measured as a function of temperature. These relationships are linear in the range of operation for the PEEK plastic. The speed of sound in PMMA has a slight curvature above $25^{\circ} \mathrm{C}$ (Fig. 4). With the probe assembled, the attenuation in BR2 was measured with both air and water as samples. $\alpha_{2}$ was estimated using (5) and the known densities of the samples. Fig. 4 shows the linear cumulative attenuation $\alpha_{2}$ at $2.0 \mathrm{MHz}$ over the temperature range investigated.

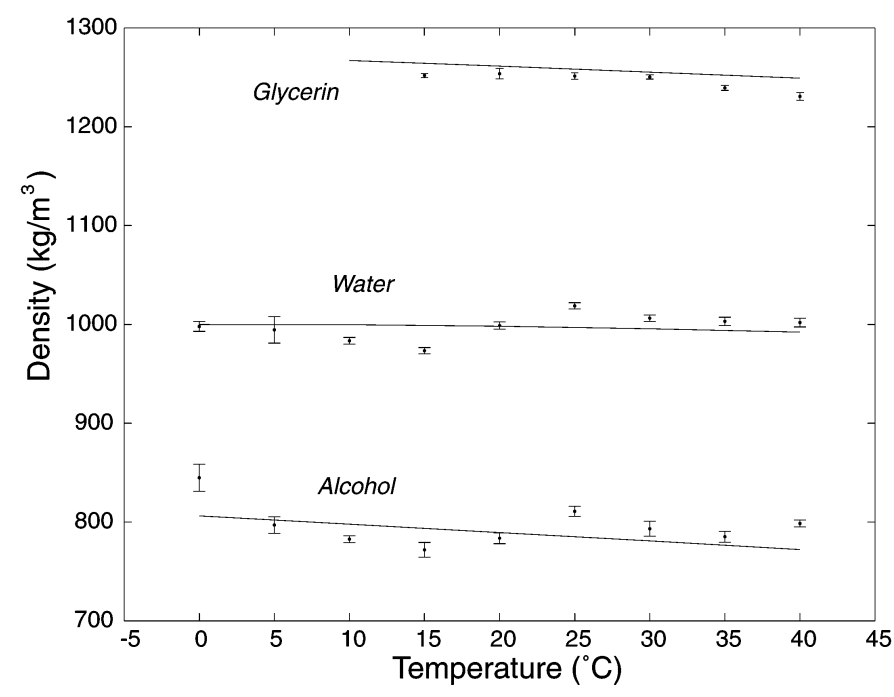

Fig. 6. Calculated densities of three liquids versus tabulated data. The $95 \%$ confidence intervals are indicated for all measured values.

\section{Thermal Steady State}

1. Density Measurements: After a minimum of $3 \mathrm{~h}$ at a constant temperature, the system reaches a state at which its temperature varies no more than $0.2^{\circ} \mathrm{C}$. One hundred measurements are taken over a 15-min interval at each temperature step. The temperature ranges are between 0 and $40^{\circ} \mathrm{C}$ for $99 \%$ pure ethyl alcohol; 2 and $40^{\circ} \mathrm{C}$ for degassed, purified water; and 15 and $40^{\circ} \mathrm{C}$ for $99.5 \%$ waterfree glycerin. The temperature steps are $5^{\circ} \mathrm{C}$. The lower limit for water is set to prevent freezing. A loss of signal imposes the lower limit for glycerin. Below $15^{\circ} \mathrm{C}$, the acoustic attenuation in glycerin becomes so large that the third echo becomes undifferentiable from noise, thus rendering the bulk speed of sound in glycerin unobtainable.

Fig. 6 depicts the average of the measurements at each temperature. The solid curves, used as reference, are tabulated values from [12] for water and from [13] for alcohol and glycerin. Two types of errors are distinguishable: a random error around the set averages and a fixed and replicable error between the averages and the references. The error bars around each measurement show that, in general, the random uncertainty is less than $0.5 \%$. The following error analysis section discusses this uncertainty. The second part of the error analysis section covers the oscillating pattern of the fixed error, which reaches a maximum value of $2 \%$.

2. Error Analysis: The uncertainty analysis of the densitometer system provides a picture of which measurement contributes the most to the uncertainty of the density estimation. A general uncertainty analysis is chosen for the following two reasons. First, the operating ranges of temperatures and liquid densities are wide, such that the bias or fixed error at each operating point becomes random over the whole ranges of densities and temperatures. For example, consider the acoustic attenuation in BR2. The amplitude of the second echo changes drastically from 0 


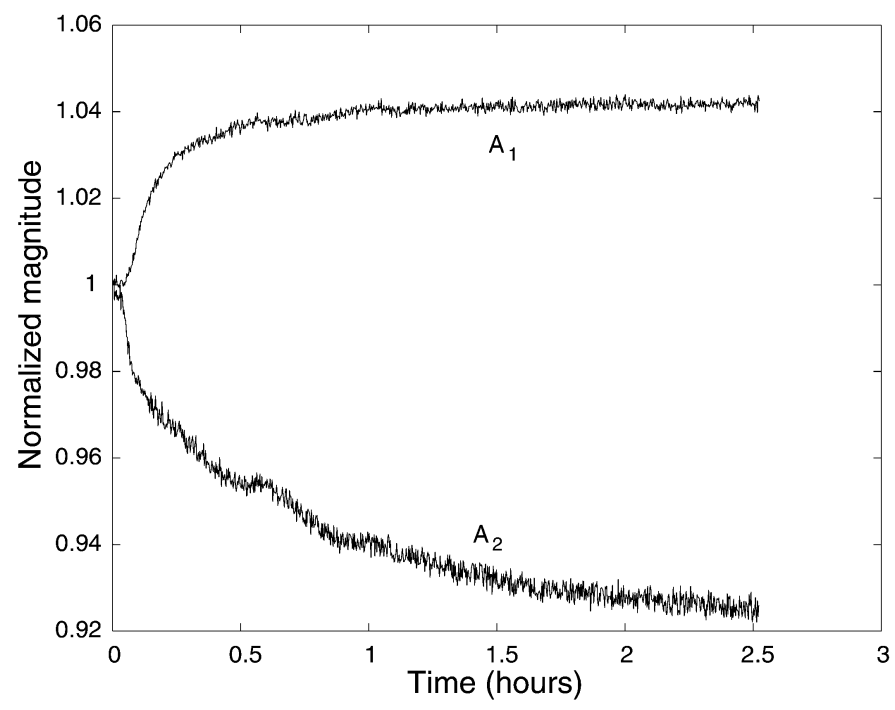

Fig. 7. Normalized amplitude of the received echoes during the thermal step change of 20 to $25^{\circ} \mathrm{C}$.

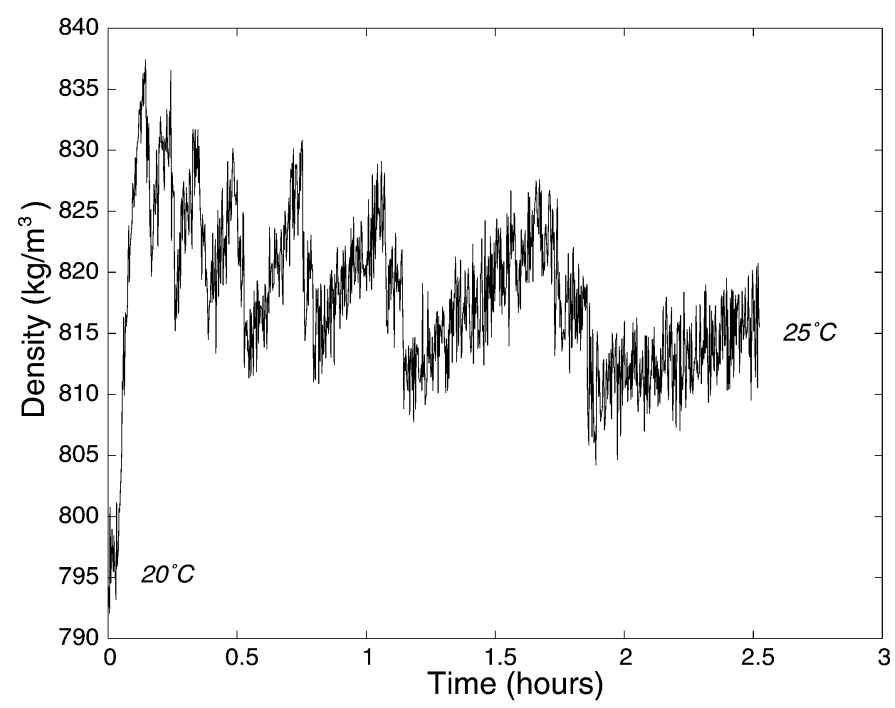

Fig. 8. Calculated densities of alcohol from 20 to $25^{\circ} \mathrm{C}$ corrected for a thermal gradient across BR2.

and $40^{\circ} \mathrm{C}$ because of a near doubling of the attenuation coefficient (Figs. 4 and 7). Secondly, the major correlation between the measurements is temperature, as is shown in the thermal transient section (Fig. 8).

The general uncertainty analysis is based on (5), and the probe is as described earlier. The uncertainty $U_{\rho_{3}}$ of the liquid's density is calculated as

$$
U_{\rho_{3}}=\left[\sum_{i=1}^{11}\left(\frac{\partial \rho_{3}}{\partial x_{i}} U_{x_{i}}\right)^{2}\right]^{\frac{1}{2}}
$$

where $x_{i}$ refers to the variables needed to calculate the density $\rho_{3}$. The partial derivatives $\partial \rho_{3} / \partial x_{i}$ provide the sensitivity of the density calculation to the respective variables. Table I lists the variables involved, the sensitivity of $\rho_{3}$ to each of them, the estimated uncertainty with a $95 \%$
TABLE I

Uncertainty Analysis of the Probe with Water.

\begin{tabular}{cccc}
\hline$x_{i}$ & $\partial \rho_{3} / \partial x_{i}$ & $U_{x_{i}}$ & $\left|\left(\partial \rho_{3} / \partial x_{i}\right) U_{x_{i}}\right|$ \\
\hline$\rho_{1}$ & -11.29 & $0.060 \mathrm{~kg} / \mathrm{m}^{3}$ & 0.68 \\
$d_{1}$ & $-595.91 \cdot 10^{3}$ & $1 \mu \mathrm{m}$ & 0.60 \\
$t_{1}$ & $388.57 \cdot 10^{6}$ & $5 \mathrm{~ns}$ & 1.94 \\
$\rho_{2}$ & 13.59 & $0.050 \mathrm{~kg} / \mathrm{m}^{3}$ & 0.68 \\
$d_{2}$ & $717.19 \cdot 10^{3}$ & $1 \mu \mathrm{m}$ & 0.72 \\
$t_{2}$ & $-553.93 \cdot 10^{6}$ & $5 \mathrm{~ns}$ & 2.77 \\
$\alpha_{2}$ & -34.46 & $0.1 \mathrm{~Np} / \mathrm{m}$ & 3.45 \\
$d_{3}$ & $-33.48 \cdot 10^{3}$ & $1 \mu \mathrm{m}$ & 0.07 \\
$t_{3}$ & $12.41 \cdot 10^{6}$ & $5 \mathrm{~ns}$ & 0.06 \\
$A_{1}$ & $0.046 \cdot 10^{6}$ & $50 \mu \mathrm{V}$ & 2.31 \\
$A_{2}$ & $-0.023 \cdot 10^{6}$ & $150 \mu \mathrm{V}$ & 3.50 \\
$\rho_{3}$ & - & $6.49 \mathrm{~kg} / \mathrm{m}^{3}$ & - \\
\hline
\end{tabular}

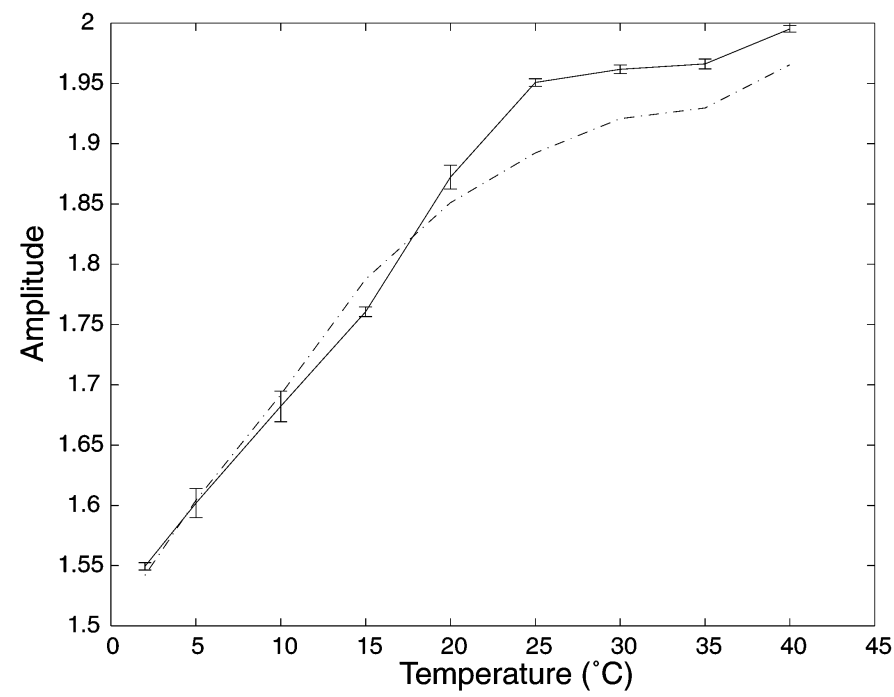

Fig. 9. Measured (solid) and anticipated amplitude of the reference echo at $2 \mathrm{MHz}$ as a function of temperature.

confidence level associated with each variable, and the absolute value of their product. The liquid chosen was water at a temperature of $20^{\circ} \mathrm{C}$.

The analysis indicates that an accuracy $0.65 \%$ is achievable. By reducing the sampling delay to $1 \mathrm{~ns}$, which is readily achievable with today's electronics, and with it the uncertainty of the other thermally associated variables, an accuracy of $0.4 \%$ is attainable. This is confirmed by the uncertainty around the mean of each of the hundred measurement sets. It should be noted here that the distance to the reflector $d_{3}$ is assumed to be constant, regardless of the temperature, creating a maximum bias error of $0.1 \%$ in the calculation of the speed of sound in the liquid.

To better understand the oscillating fixed error around the reference curves (Fig. 6), all the variables involved in the estimation of $\rho_{3}$ were analyzed. The amplitude $A_{1}$ versus temperature shows an unexpected change of slope at room temperature (Fig. 9). Using the reference density data along with the other measured values at the various temperatures, a theoretically anticipated amplitude $A_{1}$ is derived using (5). In Fig. 9, the measured amplitude $A_{1}$ is 


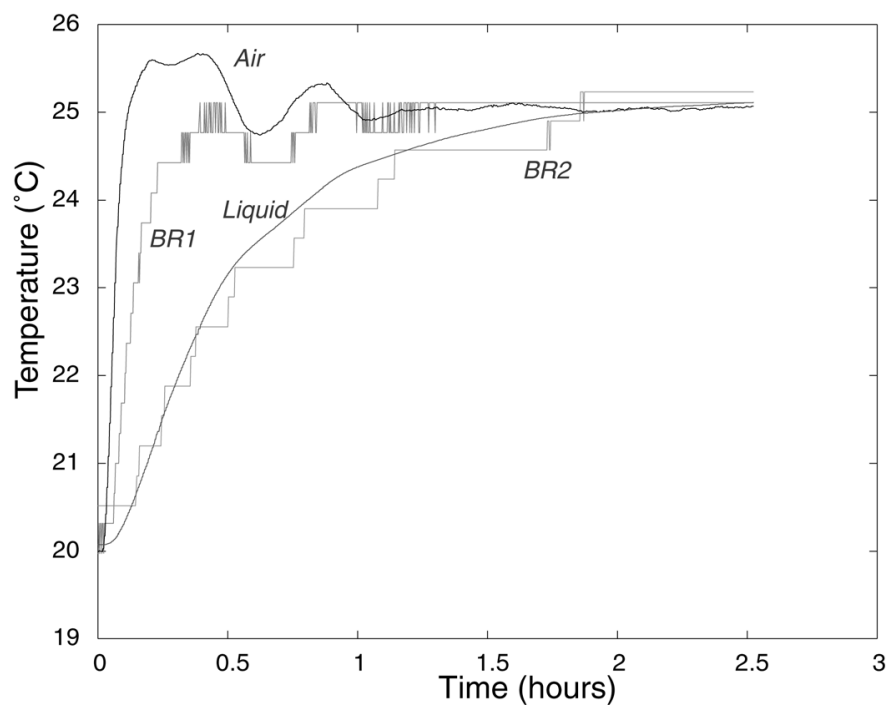

Fig. 10. Measured temperature of the environment and the alcohol. Estimated temperatures of BR1 and BR2.

compared with the anticipated amplitude $A_{1}$. The figure reveals that the coefficient of reflection at that interface deviates from the proposed theory. The received echoes are smaller than the anticipated ones at lower temperatures and larger at higher temperatures. This is potentially caused by the different coefficients of thermal expansion for the two buffer rods and the cyanoacrylate layer and is a violation of the requirement of set by (1) and (4) of a flat interface. This effect can be masked by a non-linear coefficient of attenuation $\alpha_{2}$ with respect to temperature.

\section{Thermal Transient}

Density measurements of liquids taken while the temperature is changing show some interesting characteristics of the probe. The temperature sweep forms a thermal gradient that alters the material properties along the probe and highlights the limitation of the device caused by an insufficient sampling rate.

Fig. 10 shows the measured and estimated temperatures during a thermal step change from 20 to $25^{\circ} \mathrm{C}$. The temperatures of the chamber's air and liquid are measured using the PT100 probes. The two stair-step-like temperature curves depict the estimated temperature based on speed of sound in BR1 and BR2. The temperature is held constant until the change in time of flight is detected. As the sampling period is $5 \mathrm{~ns}$, the temperature estimate is limited to a resolution of about one-third of a degree.

With a thermal gradient, the acoustic impedances vary throughout the buffer rods, and (5) needs to be revised to account for this. Introducing $z_{2 a}$ and $z_{2 b}$ as the acoustic impedances of PMMA at the BR1-BR2 interface and at the BR2-liquid interface, respectively, (5) becomes

$$
\rho_{3}=\frac{z_{2 b}}{c_{3}} \frac{\left(4 A_{1} z_{1} z_{2 a} e^{-2 d_{2} \alpha_{2}}-A_{2}\left(z_{1}^{2}-z_{2 a}^{2}\right)\right)}{\left(4 A_{1} z_{1} z_{2 a} e^{-2 d_{2} \alpha_{2}}+A_{2}\left(z_{1}^{2}-z_{2 a}^{2}\right)\right)} .
$$

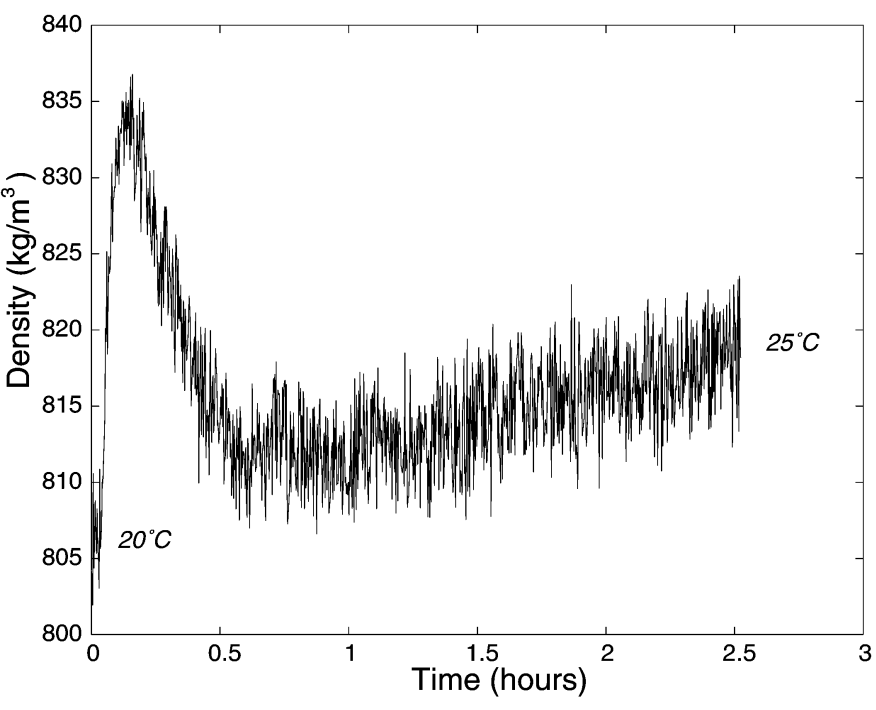

Fig. 11. Calculated density of alcohol from 20 to $25^{\circ} \mathrm{C}$ with thermal gradient correction and high resolution thermal measurements.

The acoustic impedances and acoustic attenuation of this new equation need the correct temperature estimate. Referring to Fig. 10, it is clear that the temperature of BR2 follows the liquid's temperature with a minor phase delay. The BR2-estimated temperature is a good candidate to calculate the correct acoustic impedance $z_{2 b}$ at the BR2liquid interface. Fig. 7 traces the amplitude of the first two echoes $A_{1}$ and $A_{2}$ measured during the thermal transient. Comparing Fig. 7 and 10, the amplitude $A_{1}$ emulates the temperature of BR1. This presents the temperature estimated from the speed of sound in BR1 as an obvious choice to obtain the correct acoustic impedances at the BR1-BR2 interface $\left(z_{1}\right.$ and $\left.z_{2 a}\right)$. The attenuation $\alpha_{2}$ uses the thermal estimate of BR2 as it is based on the average speed of sound through BR2. Fig. 8 shows the density calculations of alcohol during a thermal change from 20 to $25^{\circ} \mathrm{C}$. If the presence of the thermal gradient is not taken into account, the error reaches $20 \%$ during this small temperature change.

A saw tooth pattern appears in Fig. 8. The sharp corrections to the density measurement (1\% per correction) correspond to step increases in the estimated temperature of BR2 (Fig. 10). As with the uncertainty analysis, it is clear that the sampling time of $5 \mathrm{~ns}$ is not sufficient. This is solvable by using high-speed acquisition equipment or a subsampling estimation technique as proposed by Grennberg and Sandell [14]. To simulate a higher resolution of temperature estimation, the measured temperature of the liquid is used instead of the estimate from BR2. The resulting density measurements (Fig. 11) form a smoother transition from 20 to $25^{\circ} \mathrm{C}$.

The density measurement deviates during the first half hour of thermal transient from a straight line connecting the start and end points (Fig. 11). Analysis of the measured signals reveals a potential origin. The amplitude of the second echo, $A_{2}$, decreases very rapidly at the beginning of the thermal step change (Fig. 7), much faster than 
the increase of the estimated temperature of BR2. After the first half hour, the amplitude $A_{2}$ holds constant until it catches up with the loss associated with $\alpha_{2}$ as a function of the estimated temperature in BR2. It is now speculated, without proof, to be related to the coupling cyanoacrylate layer between the two buffer rods. This speculation is based on the fact that the anomaly in $A_{2}$ disappears when the temperature of the reference interface stabilizes (Fig. 10).

\section{CONCLusion}

The study presents a density probe capable of measuring liquid densities of 750 to $1300 \mathrm{~kg} / \mathrm{m}^{3}$ at constant temperatures ranging from 0 to $40^{\circ} \mathrm{C}$. The uncertainty with a $95 \%$ confidence level is $2.5 \%$ for those measurements done in a thermally static environment. As much as $1.0 \%$ is due to an insufficient time resolution in the speed of sound measurement. The error analysis shows that a density measurement of $0.4 \%$ accuracy is possible.

During thermal transients, a thermal gradient is developed along the probe, which directly influences the characteristic impedances throughout the probe. This issue is handled by correctly assessing the temperature at each interface.

Both in thermally static and thermally dynamic situations, the interface between the two buffer rods seems to influence the accuracy of the probe. The different thermal expansion of the polymer and thermodynamics of the thin glue layer may play a role that needs to be included in the model. Further investigations are necessary to develop a better understanding.

Returning to the industrial perspective of the introduction, the current probe is inadequate to track thermal changes in normal liquids. However, the probe is suitable for the process industry if the thermal changes are slow. Examples include the mixing of water and glycerin or the fermenting of a fruit juice in which 10 to $12 \%$ of its volume turns to alcohol.

\section{REFERENCES}

[1] D. W. Spitzer, Ed. Flow Measurement. Research Triangle Park, NC: Instrum. Soc. Amer., 1991.

[2] L. E. Kinsler, A. R. Frey, A. B. Coppens, and J. V. Sanders, Fundamentals of Acoustics. 3rd ed. New York, NY: John Wiley \& Sons, 1982.

[3] J. Kritz, "Continuously indicating electroacoustic densitometer," U.S. Patent $2869357,1959$.
[4] J. Kritz, "Mass flow fluid measurement," U.S. Patent 2911825 , 1959.

[5] V. A. Sukatskas, V. K. Armoshka, and E. V. Stankyavichyus, "Ultrasonic densimeter for liquids," Meas. Tech., vol. 36, no. 3, pp. 44-45, Mar. 1993.

[6] J. C. Adamowski, F. Buiochi, C. Simon, and E.C.N. Silva, "Ultrasonic measurement of density of liquids," J. Acoust. Soc. Amer., vol. 97, no. 1, pp. 354-361, Jan. 1995.

[7] A. R. Guilbert and M. L. Sanderson, "A high accuracy ultrasonic mass flowmeter for liquids," in FLOMEKO 96 Proc., pp. 244249 .

[8] A. Püttmer, "Ultrasonic density sensor for liquids," Ph.D. dissertation, Aachen: Shaker Verlag, 1999.

[9] J. Delsing, "A new type of ultrasonic densitometer," Ph.D dissertation, Lund Institute of Technology, Lund, Sweden, 1988.

[10] J. A. van Deventer and J. Delsing, "An ultrasonic density probe," in Proc. 1997 IEEE Ultrason. Symp., pp. 871-875.

[11] W. H. Press, S. A. Teukolsky, W. T. Vetterling, and B. P. Flannery, Numerical Recipes in C. 2nd ed. Cambridge, UK: Cambridge University Press, 1992.

[12] G.W.C. Kaye and T. H. Laby, Tables of Physical and Chemical Constants. 14th ed. London, UK: Longman Group Limited, 1973.

[13] E. B. Freyer, J. C. Freyer, and D. H. Andrews, "Sonic studies of the physical properties of liquids. I. The sonic interferometer. The velocity of sound in some organic liquids and their compressibilities," J. Amer. Chem. Soc., vol. 51, pp. 244-249, Mar. 1929.

[14] A. Grennberg and M. Sandell, "Estimation of subsample time delay differences in narrowband ultrasonic echoes using the Hilbert transform correlation," IEEE Trans. Ultrason., Ferroelect., Freq. Contr., vol. 41, no. 5, pp. 588-595, 1994.

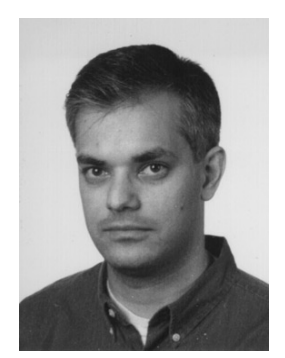

Jan A. van Deventer was born on September 15, 1965. He received his B.S. in physics from Hope College, Holland, MI, in 1987 and his M.S. in mechanical engineering from the University of Michigan, Ann Arbor, MI in 1989. From 1991 to 1996, he has worked worldwide with Kelsey-Hayes in the development of anti-locking braking systems. Since 1996, he has been at the division of Industrial Electronics at Luleå University of Technology as a Ph.D. student. His research interest is the development of an ultrasonic mass flowmeter.

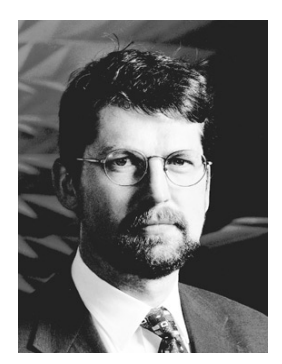

Jerker Delsing (S'85-M'88) was born in Umeå, Sweden on June 3, 1957. He received the M.Sc. degree in engineering physics from the Lund Institute of Technology, Sweden in 1982. In 1985, he received his Ph.D. in electrical measurement technology at Lund Institute of Technology. In 1995, he was appointed professor in industrial electronics at Luleå University of Technology, Sweden. Areas of research interest are ultrasonic measurements, EMC, and embedded Internet systems. Professor Delsing is chairman of the Instrument Society of Sweden. 